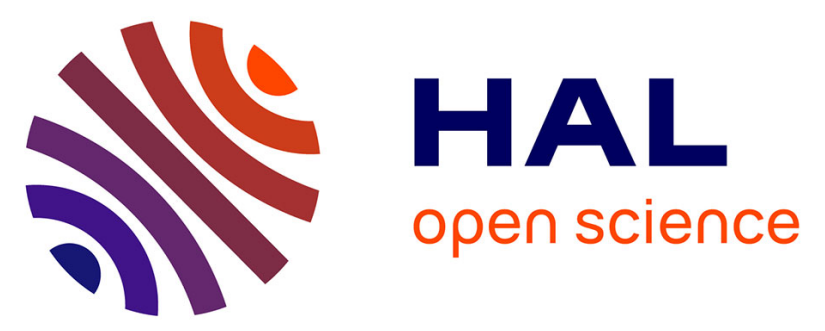

\title{
Designing Wiimprovisation for Mediation in Group Music Therapy with Children Suffering from Behavioral Disorders
}

Samuel Benveniste, Pierre Jouvelot, Edith Lecourt, Renaud Michel

\section{- To cite this version:}

Samuel Benveniste, Pierre Jouvelot, Edith Lecourt, Renaud Michel. Designing Wiimprovisation for Mediation in Group Music Therapy with Children Suffering from Behavioral Disorders. the 8th International Conference on Interaction Design and Children, Jun 2009, Como, France. pp. 18-26, 10.1145/1551788.1551793 . hal-00917975

HAL Id: hal-00917975

https://hal-mines-paristech.archives-ouvertes.fr/hal-00917975

Submitted on 12 Dec 2013

HAL is a multi-disciplinary open access archive for the deposit and dissemination of scientific research documents, whether they are published or not. The documents may come from teaching and research institutions in France or abroad, or from public or private research centers.
L'archive ouverte pluridisciplinaire $\mathbf{H A L}$, est destinée au dépôt et à la diffusion de documents scientifiques de niveau recherche, publiés ou non, émanant des établissements d'enseignement et de recherche français ou étrangers, des laboratoires publics ou privés. 


\section{Designing Wiimprovisation for Mediation in Group Music Therapy with Children Suffering from Behavioral Disorders}

\author{
Samuel Benveniste, Pierre Jouvelot \\ $\mathrm{CRI}$ - Centre de recherche en informatique \\ Mathématiques et systèmes \\ MINES ParisTech \\ 35 rue Saint-Honoré \\ 77305 Fontainebleau Cedex, France
}

\author{
Edith Lecourt, Renaud Michel \\ Laboratoire LPCP 4056 EA \\ Université Paris Descartes \\ 71 avenue Edouard Vaillant \\ 92774 Boulogne Billancourt Cedex, France
}

\begin{abstract}
We present qualitative experimental evidence that the "Wiimprovisation" process of using Nintendo Wiimotes as virtual instruments linked to a musical sound system within the framework of Group Music Therapy sessions can provide effective psychodynamic mediation for patients. We designed and implemented specific modalities for movement-based sound control and tested our tool in two clinical settings with children (age 7 to 12) suffering from behavioral disorders. Our preliminary results show immediate acceptance of, long term motivation for and meaningful expression through our system by patients. Future work will focus on assessing the therapeutic potential of our platform's personalization features in a controlled experiment.
\end{abstract}

\section{Categories and Subject Descriptors}

J.3 [Life and Medical Sciences]: Health; J.5 [Arts and Humanities]: Performing Arts---music; H.5.2 [Information Interfaces and Presentation]: User Interfaces---user-centered design; K.8.0 [Personal Computing]: General---games.

\section{General Terms}

Design, Experimentation, Human Factors

\section{Keywords}

Mediation, video game, Wiimote, music therapy

\section{INTRODUCTION}

Music Therapy [8] is a therapeutic practice that uses music as a communication medium between patients and therapists. This ageold technique, endorsed by Plato as well as ancient Chinese healers, has been put to good use by a large group of practitioners, gathered into professional associations such as the American Music Therapy Association [2] or the French "Association française de musicothérapie" [4]. There exist two main Music

\footnotetext{
Permission to make digital or hard copies of all or part of this work for personal or classroom use is granted without fee provided that copies are not made or distributed for profit or commercial advantage and that copies bear this notice and the full citation on the first page. To copy otherwise, or republish, to post on servers or to redistribute to lists, requires prior specific permission and/or a fee.
}

IDC 2009, June 3-5, 2009, Como, Italy

Copyright 2009 ACM 978-1-60558-395-2/10/08... \$5.00.
Therapy variants: (1) Receptive Music Therapy [8], where patients listen to carefully chosen pieces of recorded music, and which can help in stress and pain management, for instance in hospitals and nursing homes [1], and (2) Active Music Therapy [9], where clients (and possibly therapists) play instruments to create musical performances, which can be organized for groups or single patients.

One of the goals of Active Music Therapy processes is to induce mediations. Mediation [10] is the psychological process in which patients undertaking psychodynamic therapy take advantage of an object they can link, although sometimes not in a clear and direct way, to their personal histories to express meaningful behaviors or feelings. Here, for instance, a patient might find in the "roundness" of a drum sound an echo of a former situation he or she experienced and that lead to his or her current traumatic condition.

Even though traditional musical instruments have proven to be conducive to effective mediation in various settings [18], we report in this paper that more advanced technologies can not only be effective for mediation purposes, in particular for children and young adults who are more familiar with technological devices, but also provide significant advantages to the music therapy process. We designed and implemented MAWii, a music therapy system that links Nintendo Wiimotes to a sound generation system. We tested it in a therapeutic setting by letting young patients "Wiimprovise", a name we coined to denote the use of Wiimotes in music production for therapeutic purposes according to (a small variant of) the Sonorous Communication framework [18], a general protocol for Group Music Therapy. Our results suggest that our video game-based virtual instruments indeed support mediation, while enabling the derivation of additional benefits in terms of motivation, personalization, data analysis and logistics.

The structure of this paper is as follows. In "Related Work", we review the relevant literature, which pertains to both the video game and music therapy domains. We then describe our general framework and its expected benefits in "Wiimprovisation". In "Experimental Conditions", we review the validation and longrange field tests we organized to assess the mediation capabilities of our system, the details of which are provided in "System Design": we put there the emphasis on the design process that lead to the current prototype. In "Results", we describe the psychodynamic observations that allow us to assert that our 
system provides the mediation capabilities required for Group Music Therapy. Finally, we summarize our results in the "Conclusion".

\section{RELATED WORK}

If several promising systems such as MIDICreator [14] have already been desgined for music therapy, we found no mentions in the literature of a system specifically tailored to enhance group music therapy with a video game flavor. However, our approach draws upon previous research projects blending video games with health care or musical expression in a great variety of ways.

\subsection{Music Games}

Music games have thrived these past few years, mainly thanks to the new control interfaces that are now available to the public at an affordable price.

Guitar Hero and Rock Band use specific devices that can be seen as simplified and stylized versions of a guitar, drum set, etc. The gameplay is very straightforward: some kind of musical score scrolls through the screen and the player is ranked on his/her ability to execute the required synchronized presses, strokes etc. with adequate timing. These games put emphasis not on gameplay but on making the player feel like s/he is a true virtuoso rock musician. Their enormous commercial success clearly shows that an intricate gameplay is not necessary to motivate players, since things such as hard challenge and strong evocative power can be sufficient.

Wii Music invites the player to try four different instrument types, each with its specific Wiimote control gestures. The game puts much more emphasis on experimentation and improvisation. Though such a sandbox approach may not work with teenagers thrilled by challenge, as shown by comments on video game websites such as [15], younger children and older, casual or even non-gamers are much more interested in this kind of gameplay, as was shown with Pads'n'Swing [11], a serious game where the underlying motto was jazz improvisation teaching.

\subsection{Health and Video Games}

In his various papers (see [21] for an example), Michael Stora describes how he uses several carefully chosen off-the-shelf video games as support for his work with children in analytical psychotherapy. He has shown that video games with strong narrative content such as Fable or Ico can be very efficient and compelling mediation objects for children who have difficulties to talk about their suffering. It enables them to symbolically dive back into the traumatic situations that caused their trouble and learn to deal with them in a reassuring setting with the therapist's help.

In work that has yet to be published, but has received extensive media coverage, Kahol and Smith [16] study the benefits for surgeons of playing Marble Mania on the Wii. The fine motor skills that are put to the test in the game (Wiimote pitch and roll control) are, according to them, very similar to those required to perform clean and efficient surgery. Results suggest that surgeons playing one hour of Marble Mania a day perform $48 \%$ better than those who do not. This means that the Wiimote is sensitive and precise enough to qualify as a complement to, or maybe even as a replacement of, medical simulators which often cost tens of thousands of dollars.
WiiFit provides a new and fun way to exercise by offering the player various games that are played with the Wii Balance Board. These are divided into four categories: strength training, aerobics, yoga and balance games. Besides raising awareness on the dangers of too little exercise, WiiFit is more of an exergame. The gameplay is what is truly beneficial: playing is fun but requires an effort and the automatic scoring and tracking through time motivates the player to exercise every day.

\subsection{Serious Games for Health}

The number of patients in need for functional reeducation is higher than ever, most notably due to a significant increase in survival rates for strokes and accidents in general. However, although efficient if carried out properly, rehabilitation is a tedious process that often fails because patients drop treatment. This is a textbook case for the application of serious gaming techniques, as shown by the ongoing Rehab Games project at the University of Skövde [5]. For example, a simple interface with pull ropes, much like a rowing machine, was designed for upper body rehabilitation. From a motor point of view, patients exercise just the same as they usually do. But a gaming layer is superimposed on this process: the patient's movements may result in bad guys getting punched, a bike steered out of danger etc. As with WiiFit, rehabilitation becomes fun and patients therefore commit to the treatment more willingly.

Remission [13] intends to improve treatment acceptance in children undergoing chemotherapy. It takes the form of a third person shooter game where the player has to wander in a child's body and destroy cancerous cells. Throughout the game the player learns about the disease and how the treatment works. Playing Remission has been shown to improve the children's knowledge and self-esteem, which in turn leads to better commitment to the treatment. As the term learning game indicates, what is beneficial here is the knowledge gained by the player. The gameplay in itself is absolutely standard and only serves as a catalyst.

The precise tracking of a patient's progress via video game scores becomes possible and can serve both as a motivation for the patient and as an assessment tool for therapists. This calls for systems able to keep track of the large amount of data that is generated, such as the MT Logbook, a computer environment geared towards music therapists and developed by the University of York [22].

\section{WIIMPROVISATION}

Wiimprovisation is a therapeutic process we designed to build upon these previous ideas so as to enhance psychodynamic therapy in a musical setting. It introduces Wiimotes as virtual instruments in a more traditional musical mediation therapy.

\subsection{Mediation in Sonorous Communication}

In psychotherapy, the term mediation, as defined by Chouvier [10], refers to any auxiliary activity supervised by the therapist in order to encourage patients to talk about personal issues that would otherwise remain untold. This concept is useful in the analysis of a large number of techniques such as play therapy, role playing or art therapy.

Lecourt's Sonorous Communication [18], a music therapy framework widely used in France, sees free group improvisation as a therapeutic mediation. Although designed independently, Sonorous Communication exhibits similarities with Priestley's 
Analytical Music Therapy [9], which is also based on Freudian psychodynamic principles, and Creative Music Therapy [9], created by Nordoff and Robbins, which focuses on the use of musical improvisation too. However, it differs from these two approaches by targeting group mediation, while Priestley emphasizes one-to-one therapist-client connections, and not using music education as a therapeutic means, whereas improving patients' musical skills is one of the main goals of the NordoffRobbins method.

In Sonorous Communication, patients are presented with a set of instruments chosen by the therapist and they may pick one or more each. They may also choose to simply participate with their voice or perform percussions with their body or with any kind of device that produces sound. Patients are then invited to play in groups ranging from 2 to 10 persons while the therapist steps back to watch, and sometimes stop the improvisation after about ten minutes if the group cannot manage to come to a halt on its own.

The only suggestion given to the group is to "communicate through sound, with eyes closed if possible". This is very important: at no point during the session is musicality presented as a goal. Sounds are from the start understood as signs that convey some kind of meaning, and in this sense could be seen as closer to words. However it seems that the most beneficial parts of the improvisation are those when the players clearly feel that their production as a group [3] is "musical", and often, but not necessarily, beautiful.

Sonorous Communication includes three more phases that are considered to be equally important as the playing phase (see Figure 1): a first discussion, then a replay of the recording of the performance, and another discussion. Through the analysis of these verbalizations, it can be seen that those "musical" moments are the events that patients are the most willing to elaborate about, which is the definition of an efficient mediation object.

\begin{tabular}{|c|c|c|c|c|}
\hline $\begin{array}{c}\text { Inst. } \\
\text { choice }\end{array}$ & $\begin{array}{c}\text { Improvis } \\
\text { ation }\end{array}$ & $\begin{array}{c}\text { Verbaliz } \\
\text { ation }\end{array}$ & $\begin{array}{c}\text { Repl } \\
\text { ay }\end{array}$ & $\begin{array}{c}\text { Verbaliz } \\
\text { ation }\end{array}$ \\
\hline
\end{tabular}

\subsection{Objectives and Constraints}

The overall goal of our research is to explore the potential benefits and caveats of introducing computer and video game technology in music therapy. We think this is a worthwhile effort: children today are increasingly familiar with such technologies and psychotherapy has to follow or to be at risk of losing contact with its younger patients.

Given the widespread skepticism of music therapists towards computer technologies [23], a first step is to show the feasibility of such an approach, i.e., that a computer-based system can be used in an actual clinical setting and perform the same role of mediation object as traditional instruments without any significant modification of the usual protocol. These requirements stem from two major objections often made by music therapists.

Firstly, non tech-savvy health care professionals must be able to configure and use computer systems with patients who require constant attention and easily give up if something does not work properly right away. Hence the requirement for our system to be easy to use, but most importantly very robust, so as not to lose the patients' attention with technical failures. This need becomes even more acute because engineers and technicians are often not allowed to enter health care institutions. Though the need for their actual presence in the clinical universe is increasingly acknowledged, ethical and safety concerns might always prevent them from being there to fix things on the spot.

The second, more profound issue is the validity of artificial interfaces as mediation objects, despite their lack of corporality. Indeed, compared to traditional instruments, affordable devices such as the Wiimote have a plastic feel and produce their sound through distant speakers instead of vibrating locally. Music therapists remain skeptical about the capability of such systems to trigger a true emotional and creative response from patients. Therefore, another goal of our experiments is to show that properly designed artificial interfaces can retain enough emotional charge and musical expressiveness to be used in combination with or even instead of traditional instruments. Otherwise it will be impossible to leverage the numerous extra possibilities they offer, however useful they might be.

\subsection{Potential Benefits}

To make a "business case" for the introduction of Wiimprovisation in a therapeutic setting, an objective assessment of the many possible positive impacts of this framework needs to be made.

We aim at properly leveraging the great motivational power of video games to improve patient commitment. This is not as straightforward as it sounds since, as explained by G. Denis in his study of the game Pads'n'Swing [11], not all motivating aspects of video games are compatible with creativity, which is very important in music therapy and absolutely central in Sonorous Communication. However, we think that the aura of fun that devices like the Wiimote carry can greatly appeal to children and motivate them to better focus during their work with their therapist.

With a proper interface, instruments combining virtually any conceivable gestures and sounds can be created. Therefore, video game technology now gives us the ability to personalize each client's instrument according to his/her wishes. The most obvious advantage of such a capability is the possibility to adapt the instrument to the client's skills and, if necessary, his/her disabilities. This means that a patient who does not know how to or even physically cannot play guitar may still be given the possibility to use the guitar sound $\mathrm{s} / \mathrm{he}$ loves.

A less obvious, but in our opinion maybe even greater benefit, is the possibility for the therapist to progressively build a custom instrument with each patient. We think this would be an excellent way to maintain interest and improve patients' self-esteem by accompanying progress and rewarding mastery with new capabilities and personal sounds, very much like what is done in video games. From a therapist's perspective, such a process could also be used to assess a patient's evolution. For example, music therapists pay a lot of attention to the choice of instruments a patient makes, since it can often indicate underlying psychodynamic changes. By exploring different possibilities with their clients, therapists could draw a sort of "dynamical map" of their musical tastes in order to better reach them.

Using computer technology enables us to easily record a great deal of information during each session. By using MIDI instruments we can record each patient on a separate track for precise analysis. This would be impossible otherwise, since multi- 
track audio recording is too impractical for a clinical setting and source separation on a polyphonic track remains largely unfeasible at the moment (see Zhou [24] for a survey of possible approaches).

Also, movement information can be recorded through the devices. This is a new kind of data that has received little to no attention from therapists up to now though it is, in our opinion, very relevant. Just as plucking a string in very different ways may end up producing indistinguishable notes, many different gestures may map to the same sound. Therefore relevant changes that may be unnoticeable on the MIDI track could appear in the movement logs.

All this data, plus patient information such as which sounds were chosen at what time, can be managed and analyzed with tools like the MT Logbook [22]. For example, specific parameters such as mean volume and note density have been shown to strongly correlate with patient commitment [12]. Automatic analysis could therefore be used to segment improvisations and point to the parts most likely to be interesting, where patients are absorbed in their music.

Previous systems did perform well in a research setting but rarely made it into the hospital, mostly because they were too complicated to set up. We think that, by using simple, affordable and widespread technologies, our system could better penetrate into the clinical world and make it a lot easier, from a logistic point of view, to offer music therapy in a wide variety of institutions. Indeed, up to now, music therapists have had to travel in vans full of expensive and fragile instruments to do their job. On the contrary, a full-fledged Wiimprovisation system can fit into a small suitcase and bring music therapy where it has never been yet.

\section{EXPERIMENTAL CONDITIONS}

In this paper, we report the results of two field tests. The main one was conducted to assess the mediation capability of our Wiimprovisation process, first targeting children, whom we think will be the most receptive audience. This experiment followed a much simpler test [6], performed in Spring 2008, that validated the technical design and implementation of our system; the small system adjustments that resulted from this first experiment are described in Section "System Design".

\subsection{Setting}

These experiments took place in an after-school intensive care center where children are admitted after being diagnosed with behavioral disorders, a very broad term that may apply to a wide variety of pathologies such as hyperactivity, borderline personality disorder, instability, etc. Every evening, after school, the children are invited to participate, on a voluntary basis, in various activities organized by the staff. These for example include Lego therapy, reading sessions or relaxation.

The goal of the treatment is to help the children to learn how to better adapt themselves to a social setting. Depending on the patient's state, the kind of progress sought can vary greatly. For example, anxious, uncommunicative children will be encouraged to socialize and engage in group activities. However, they very well might quickly switch to a state of over-excitement, in response to which focus will be put on self-control, respect of the rules and of others, and on planning before acting.

\subsection{Michel's Adapted Protocol}

Sonorous Communication could not be used as is because of some specific difficulties related to the children under treatment, mainly impulsiveness, short attention span and learning difficulties. R. Michel, the therapist among us, consequently introduced four significant adjustments to Sonorous Communication.

Firstly, a welcoming phase is added at the beginning of the session. Each child in turn chooses one instrument from an album handed by the therapist instead of picking it up him/herself. This is done both to avoid conflicts and degradation of the instruments and to encourage patience and planning.

Secondly, improvisations are only meant to last for two to three minutes. After that, the children are much more likely to have difficulties to function as a group. Therefore, the therapist must be ready to stop the performance to prevent the emergence of potentially destructive processes such as violence or closing-in.

Thirdly, the instructions given to patients change from "communicating with sound" to "playing together". Emphasis is not put on communication but only on taking others into account. Indeed, the musical production of these groups can easily be very chaotic (see [7] for an audio excerpt) and detecting significant interaction between the players is already very positive, even without the additional symbolic layer that is sought after in Sonorous Communication.

Finally, due to the organization of the evenings, the children are allowed to come and go more or less as they wish. But, they have to form groups and notify the therapist in advance (during the after-school snack) that they want to come play music. Once they are done playing, the therapist is not allowed to force them to stay for the replay phases, which are carried out only with volunteers.

\subsection{Population}

As of January 2009, 15 children from age 7 to 12 are taking part in our still on-going experiments. In this paper, we draw conclusions from the results of eight weeks of testing in November and December 2008, which followed a first validation experiment held in Spring 2008.

In 18 therapy sessions, the children have tried the system in groups ranging from 1 to 3 simultaneous users. It is interesting to note that those who played alone did so because their usual partners were not present. This is why the therapist still talks about a group of 1 person, since it is therapy-wise more like a group of 3 with 2 people missing.

On average, each child used the system between three and four times. However since patients come on their own, some tried the system just once while some played up to six times. Therefore, in such a setting, a traditional test-retest validation cannot be performed, since too many parameters vary at the same time. So we decided to adopt a different approach which, in our mind, should ultimately result in a definitive design for the controlled experiment with a fixed population we are planning in the near future.

\section{SYSTEM DESIGN}

Given our unusual experiment setting, we chose to approach the design of our system using Action Research, a research methodology introduced in social sciences by Kurt Lewin [19]. Here, small changes are regularly (typically every two weeks) 
made to the ongoing design and protocol to factor in the feedback given by the therapist and children.

\subsection{Basic Architecture}

From a technical point of view, our system, called MAWii, is very similar to the setup used by live music performers, especially that of the so-called Wii-Jays. It consists of a computer hooked to a stereo amplification system through an audio interface.

A maximum of six Wiimotes, with or without extensions such as the Nunchuk, are connected to the computer via Bluetooth, while Glovepie processes the information sent, such as accelerometer measurements and button presses, and maps it to MIDI messages (see "Glovepie" below). These messages are then fed to a sequencer, Reaper, by Cockos, to record everything and drive the synthesizers.

Although this is a very standard and flexible architecture, it already contains a few clear design choices which must be justified, namely Wiimotes and Glovepie.

In the beginning, we wanted to try to use regular Playstation-like controllers to improvise, as was done with Pads'n'Swing [11]. But making music with such devices, though it can be a lot of fun, is not very intuitive and may require great cognitive efforts, beyond many music therapy patients' capabilities.

A video of WiiDrums [20] helped us realize the potential of Wiimotes. We saw that motion sensing makes it possible to design a much more straightforward interface inspired from the most intuitive instrument, the drum. Furthermore, as shown by their beneficial influence on surgeons, Wiimotes are very sensitive devices that can be used to reliably control systems with very subtle movements. This means that with proper interaction design, which will not at all necessarily mimic a real instrument, a good deal of musical expressiveness could be reached.

Carl Kenner's Glovepie [17] is a scripting language that interfaces numerous types of devices with a Windows PC (P5 Glove, Wiimote, PS3 SixAxis and so on). We use it to handle and process the data sent by the Wiimotes. It offers many useful, high level functions such as a built-in algorithm to filter out gravity in accelerometer data. With this software, complex MIDI message triggering systems can then be implemented in a rather simple way. Several classical instruments besides drums have been mimicked this way, with varying success: guitar, turntables, slide flute and probably many others.

\subsection{Proof-of-concept Design}

Before the first, validation experiment, a great deal of effort was put into designing commands that would be simple and intuitive enough for the children while retaining sufficient expressive capabilities.

\subsubsection{Triggering Mechanism}

We built upon Bob Somers' WiiDrums but modified it quite a bit to fit our needs. First, instead of using the Wiimote+Nunchuk combination, we decided to use two Wiimotes per child to keep a simple, symmetric design. More importantly, we implemented a much more complex triggering algorithm. Indeed, WiiDrums does not make any difference between the different strokes; this means that whatever force is put in, it would either do nothing or produce sounds at maximum volume, greatly impairing expressiveness.
However, interpolating the velocity of the Wiimotes is impossible with just the three-way accelerometer they carry. Therefore we chose to implement an acceleration-based triggering system. Our algorithm looks at the acceleration of the Wiimote with respect to its own $\mathrm{Y}$ axis (as labeled by Glovepie) to detect strokes and compute the volume to be played using its maximal value during the stroke. Working directly in the referential of the Wiimote is possible thanks to Glovepie's gravity filtering and gives the user some extra freedom of movement that was put to use right away, as we show below.

Interestingly, in the end, our interface does not work like a drum kit at all, since volume is determined by maximal acceleration and not by velocity on impact. Nevertheless, we thought it retained enough of a drum-like feel to remain intuitive. With a little practice it can be controlled efficiently, although traditional drum mastery will be of little help.

\subsubsection{Sound Navigation}

Obviously, one of the great advantages of the Wiimote is that it can be used to control any synthesizable sound. Therefore, instead of having just one instrument each, we gave the children the possibility to have as many instruments as they would like.

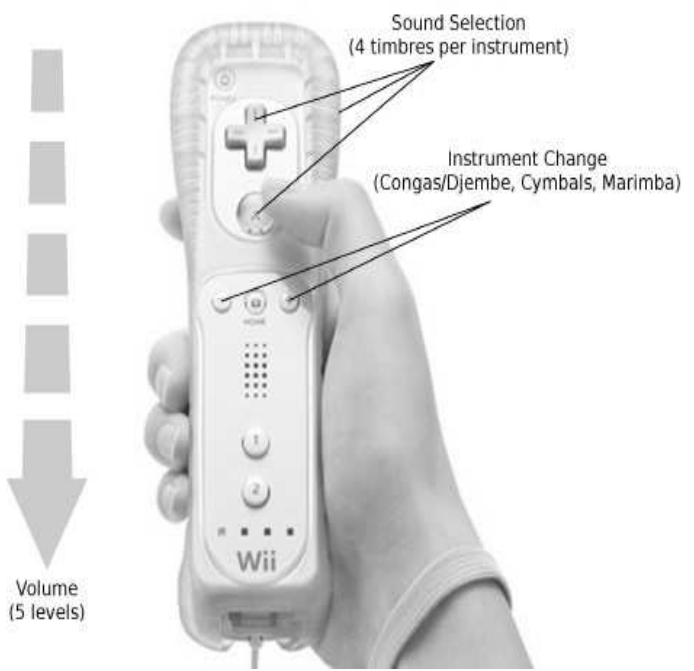

Figure 2. MAWii UI for sound generation (2 Wiimotes/patient)

For the first test, we used three instruments with four sounds each. Limiting the number of instruments, we thought, would prevent the children from getting lost when choosing. However we retained a design using the Plus and Minus buttons to cycle through the different instruments (as opposed, for example, to Buttons One and Two respectively switching to Instruments 1 and 2). This way, as many instruments as needed could be added later on while retaining the same controls (see Figure 2).

The choice of four sounds was constrained by the design of the Wiimotes. For a child's hands, only Buttons A and B (the trigger) and the directional pad seemed easily reachable. However making different directions of the D-Pad code for different sounds was too confusing, so we ended up with four sounds by not taking into account which direction was pressed on the D-Pad. Like in WiiDrums, the sound produced depends on which buttons are pressed at the instant the MIDI message is triggered. To keep a coherent design, we chose to arrange the sounds by pitch, which 
gives, in order of increasing priority: no button for the lowest pitch, lowest priority sound, B, A and finally D-pad for the highest pitch, highest priority sound.

\subsubsection{Sounds}

We wanted the instruments to have very different personalities but still fit well together. This meant that sounds that were too aggressive or too loud were not usable. And, again so as to not confuse the children, we decided to use sounds to which they had access during previous sessions with real instruments.

For this first test, we used a Roland XV-2020 MIDI synthesizer. This device works very well and reliably, but only offers a rather limited sound palette. We ended up with two percussion instruments (a conga-djembe mix and a set of cymbals) and one melodic instrument (the marimba).

\subsubsection{Protocol}

For this first technical validation experiment, we chose to give the children very little explanation about the system. The therapist had them try it out a little bit during the welcoming phase, explaining once which button did what. This is the usual approach of Sonorous Communication, which aims at putting as few constraints on creativity as possible.

However, we chose to divert a little from the standard protocol regarding the way the children were presented with our system. They were of course told that they were participating in a research experiment, as is mandatory for any clinical trial. But, following Action Research principles, the therapist implicated them further by stating that the goal was to design an instrument for them. They were encouraged to share their opinion about the system, give it a name, ask for modifications, etc.

We did this to venture into the world of personalization. Indeed, implicating the children in the design of the system is a first step towards fully customizing the instrument. We were very interested in seeing the reactions they would have, since they usually have a lot of trouble planning forward. Would the prospect of Wiimprovising again in a few months with an instrument they partly helped improve encourage them to project into the future?

\subsection{Patient-Centered Design}

The first validation test was a success [6]. It proved the soundness of our first, proof-of-concept design and the feasibility of our approach from a technical and logistic point of view while giving very encouraging signs on the motivational and psychodynamic aspects. However we also found a lot of room for improvement. Many things were modified to create a second, patient-centered version of the system taking advantage of the feedback the users (children and therapist) gave us. Here we will review this feedback and the changes it led to.

\subsubsection{Technical Changes}

For the second experiment, we switched from hardware to software, sample-based synthesizers. We used two VST plugins by Native Instruments: Battery, for percussion sounds, and Kontakt, for the rest.

Firstly, one does not need to carry an extra MIDI synthesizer anymore. With the proper ASIO drivers, any modern laptop is now powerful enough to take care of everything at a software level. Secondly, Battery and Kontakt come with a very large sample database that provides access to many more sounds than the XV-2020. Their powerful interface also makes it a lot easier to configure and tune the different sounds, add effects, compress the signals and so on.

To take advantage of this large database and give the children a broader choice of instruments, we implemented a simple GUI for the therapist. S/he can associate between one and three instruments to each pair of Wiimotes by clicking on the corresponding images. The interface then starts all the necessary software with the proper configuration.

These usability improvements are fundamental for the therapist, who already has a hard time handling the children without having to tinker with a complex computer setup. Indeed, such impulsive patients are easily distracted and lost if the therapist does not devote his full attention to them.

\subsubsection{Interface Changes}

Although the children understood the triggering mechanism right away during the first test, they had trouble with sound navigation. They did not seem to understand the difference between sound switching and instrument switching.

To make it simpler, we decided to assign a button to each instrument instead of using Plus and Minus to cycle through an arbitrarily large number of them. Minus, Home and Plus are now used to respectively switch to the first, second and third instrument.

Also, whereas the first version was very sensitive and had five volume levels available, Version 2 only offers three levels and requires quite a bit more effort to trigger the sounds. High sensitivity and large dynamic range were favored at first to enhance expressiveness and encourage the children to focus on fine motor control. However it clearly appeared during the first test that the children would not make use of such precision, and that we could trade some expressiveness for easier control.

\subsubsection{Sound Palette Exploration}

With Battery and Kontakt, it became very easy to make many more instruments available. This was one of the children's main concerns when they tried the first version. They instantly understood that virtually any sound could be built-up in the system and asked for a flute, a guitar, a piano and a derbuka.

We decided to include 6 percussive instruments and 10 melodic instruments, 3 of them using clearly synthetic sounds, for a total of 16 (one per MIDI channel). It is very hard to predict which instrument will appeal to which child, so we decided to cover a spectrum of instruments as broad as possible and see what would happen. So we blended classical percussion and melodic instruments (cymbals, congas, flute, guitar...) with traditional folklore (woodblocks, gong...) and synthetic sounds ("robot pulse", "atmospheric ambient", ...).

\subsubsection{Protocol}

We changed some aspects of the session organization in order to provide some clues about the lasting value of our system. Upon arrival, the children are now shown a slide show presenting the instruments and the sounds they make [7]. Then, they are invited to choose one instrument, and only one for now, from a booklet handed by the therapist. It contains images of all the available instruments with the real ones on one side and the MIDI ones on the other (see Figure 3). 
Unlike the first experiment, our system is given the exact same status as the usual instruments. This way we could see whether the children would still use the Wiimote system or revert to the standard instruments. Also, restricting the patients to only one instrument prevents them from being overwhelmed by the excitement induced by too large a choice.

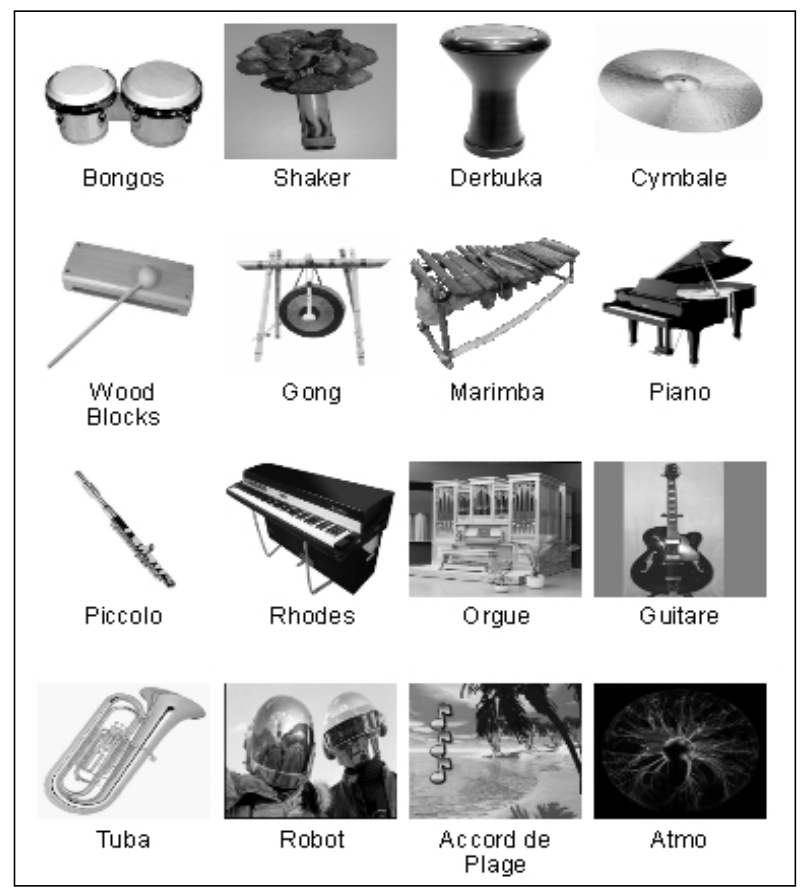

Figure 3. MIDI page of the instrument booklet

Once they have chosen their instruments, the children are invited to re-explain their understanding of how the system works. If needed, the therapist then guides them through a small tutorial, introducing the different sounds. This is the moment we intend to select in the future to describe new capabilities, such as using several instruments, to the children who start to show boredom due to increasing mastery.

\section{RESULTS}

To be an effective tool for mediation, our system must help patients to express feelings, emotions and observations about their inner self. We report below on issues such as ease-of-use, motivational and psychodynamic aspects that suggest that we indeed reached that goal.

\subsection{Ease of use and robustness}

17 out of the 18 sessions were successfully conducted and recorded with the system. Only once did it seriously crash, destroying the data of one improvisation. We therefore have shown that the system is simple and reliable enough to be used in a real clinical setting. Nonetheless, we in part owe this to the relative computer proficiency of the therapist among us, who did have to restart the software at the beginning of two sessions, due to synchronization problems in the software synthesizers.

We have also shown that our system can almost seamlessly integrate into the usual music therapy protocol. By using only hardware and software intended for the public at large, we managed to reduce costs, complexity of configuration and most importantly time consumption during the session. We thus believe that we have assembled the first music therapy system that can easily be reproduced and used as is by any therapist with a minimum of computer literacy.

\subsection{Motivation}

The second experiment confirmed the marked motivational power of our system, which the first experiment had already suggested [6], showing that the children's interest was not merely a matter of novelty. Even though they were always offered the possibility to play with a real instrument, only one of them chose to play the harmonica for one session, to set himself apart as he usually does. Otherwise they all consistently chose the Wiimotes over their former instruments. We can see two major forces at play here: the cultural aspect of video games and musicianship and the increase in self-esteem due to the children's involvement in the design process.

\subsubsection{Building Upon Cultural Trends}

The children always refer to the system as "the Wii" although there is no actual console anywhere. There is not even a screen, for that matter, but we still undoubtedly, and on purpose, take advantage of the aura of fun the Wiimotes retain. The children said things such as "It's just like on TV" or "I played the Wii, I played the Wii!" (running around telling everyone in the institution after the first session), showing that to them Wiimprovisation is very much like playing video games.

Our system also combines the aura of video games with the very positive social image of musicianship. The children for example said things like "Now I'm a real musician !".

More importantly, we think their choices of virtual instruments best reflect this cultural aspect. Indeed, we were very surprised to see that the children showed almost no interest in the percussion instruments proposed. They instead always chose to use melodic instruments, even though they had percussion-like controls. Furthermore, and again to our surprise, the children were much more attracted to classical instruments such as the guitar or piano than to the synthetic instruments we thought they would love.

This shows that we have filled two voids: (1) the lack of a melodic component to the improvisations, which seems to be central in the children's vision of music, at least in our culture, and (2) the access to the social value of musicianship, which seems to be much better incarnated by the instruments usually seen in the media (guitar, piano...) than by traditional percussion instruments, as shown by the tremendous success of games like Guitar Hero.

\subsubsection{Participative Design}

The children asked for extra instruments such as the flute or guitar after the first experiment. They also asked for colors to mark the different pairs of Wiimotes. They were obviously delighted to see that, for the second experiment, we had modified the system according to their wishes and were eager to try the new version.

It is interesting to note that, even though they never met the system designers, since, as said earlier, they are not allowed in the institution, they nevertheless are very interested in the "someone somewhere" who creates these instruments especially for them. They asked lots of questions, showing that being at the center of such a process greatly raises their self-esteem. We see this as a very strong hint that reproducing these dynamics in the future 
through instrument personalization could be a very profitable advantage of our system, on a therapeutic scale.

\subsubsection{Behavioral Benefits}

Obviously, increased motivation makes the treatment more efficient. However we would like to mention a few examples that show these benefits more precisely.

Firstly, it encourages the children to formulate projects and plan ahead, something which they often have a lot of trouble doing due to their pathologies. Indeed, even several months after the first experiment (which took place in May 2008), the children kept on talking about it and were very excited when they were told (in October) that the Wii would be back and they could use it every week this year.

Even more surprisingly, one of the most challenged patients with regard to making projects actually came to the therapist to ask for a playing slot saying: "Can we come for the Wii today with P.? We discussed this last Friday". This long term projection surprised the whole team and denotes a great deal of progress.

Secondly, better commitment to the treatment helps to deal with the various problems that may arise during sessions. The children do show a lot of excitement about the system, which is not necessarily good for a therapy intended to help them to improve self-control. However our experiments show that, thanks to their increased motivation, they are more able to focus and to control this excitement properly. This was especially apparent when the system had to be restarted during a session. Instead of losing interest, the children were actually worried that they might have broken something and remained very calm while the therapist reconfigured everything from scratch, which takes about 3 minutes. The technical failure thus turned into something positive since it encouraged the children to focus and even constituted a good topic of elaboration afterwards.

\subsection{Psychodynamic Aspects}

As mentioned above, several characteristics of our system, such as the plastic feel of the Wiimotes, have a specific psychodynamic impact that is very different from that of real instruments. However our results suggest that these differences do not prevent elaboration and that they even participate in making the system a mediation object at least as efficient as traditional instruments.

\subsubsection{Corporality}

The biggest $a$ priori objection from the music therapy community was that, due to their lack of physical presence (in terms of size, texture, vibration etc.), Wiimotes would not trigger any useful emotional response. However, this is clearly not true: our system does indeed retain enough presence to trigger meaningful elaboration and symbolization in our patient population. Moreover, its increased abstraction turns into an advantage over real instruments because it allows much more freedom of motion.

The children took advantage of this new freedom in several ways. Some got up and danced to the music while playing, which is impossible with most real instruments, resulting in much better immersion. Others started mimicking the gestures associated with the guitar sounds they were using, again resulting in better immersion and showing that the children are able to symbolically compensate for whatever lack of corporality there might be with Wiimotes.
Just as our system does not intend to rival traditional instruments in terms of musical expressiveness, since we favor ease of use, we do not aim at achieving the same richness of haptic and visual feedback. Our experiment suggests that such complex feedback, certainly a strength of traditional instruments, is not mandatory for the system to work properly as a mediation object. Therefore, we feel justified in exploring the new capabilities of our tool. It does not replace but can be used alongside traditional instruments, hopefully dragging in some previously uninterested patients and spurring new types of interactions.

\subsubsection{Identity}

Every time we used the system, even for informal tests with colleagues and "normal" children, users were concerned because they were not able to differentiate their sounds from others'. Indeed, the Wiimotes all look the same and all the sounds come out of the same remote speakers, sometimes making it very hard to know who is playing what when everyone is using the same instrument.

Regarding the look of the Wiimotes, we added color stickers and have had no complaints since then, which shows how even very little individuation can have a lot of impact. In the sound domain however, things remain complicated. The children often all choose the same instrument, making it difficult even for the therapist to follow. They often expressed their anxiety about this during sessions, but also found a solution: they stopped by themselves and started again one by one (composers of intricate pieces often use this technique to help the listener). Once more, this shows that the children are becoming able to compensate for this loss of identity, which may thus turn into an advantage, since it can trigger collaborative group processes such as those described above.

\subsubsection{Extended Sound Capabilities}

With our system, the children get access to sounds they could never produce with real instruments, which would either be too big, too expensive or too hard to play; this is very interesting from a psychodynamic point of view.

Indeed, some instruments such as the guitar, which was chosen most often, probably because of its aura, or the "atmospheric ambient" sound, a somewhat eerie artificial sound chosen by seven children out of eight on one day, carry a very strong cultural and/or emotional meaning. Our system makes it possible to build upon this to enhance mediation by offering many more sonorous universes and, consequently, emotional responses than would be possible with traditional instruments.

The best example of this is the one day mentioned above when almost all of the children in all three sessions chose the atmospheric ambient sound; since the door remains open during the sessions and the children outside can hear, this is probably not a coincidence. In all three groups, the "atmo" started a lot of discussion about fear, and more specifically about scaring someone, for real or as a joke. This clearly shows how a specific sound available only with our system may trigger a powerful group mediation process that helped the children talk extensively about potentially traumatic things they had rarely discussed before. 


\section{CONCLUSION}

Building upon Action Research rationale, we used an agile iterative process to finely design MAWii, a system that uses Wiimotes as virtual instruments, as a mediation enabler for Group Music Therapy. We report in this paper the results of two field experiments that show the extent of this mediation capability, thus validating the potential of Wiimprovisation for the treatment of psychological disorders in youngsters. This settles a fundamental question regarding the viability of our system, since mediation is a key communication requirement in psychodynamic therapies. Future work will include new designs targeting personnalization issues, controlled experimentation with a group using only traditional instruments and automatic performance data analysis.

\section{ACKNOWLEDGEMENTS}

For thank François Irigoin for his help during the preparation of this paper.

\section{REFERENCES}

[1] Aldridge, D. An Overview of Music Therapy Research. Compl.Therapies in Med., 2 (1994), 204-216.

[2] www.musictherapy.org

[3] Anzieu, D. The Group and the Unconscious. Routledge and Kegan Paul, London (UK), 1984.

[4] www.musicotherapie-afm.com

[5] Backlund, P., and students. www.his.se/forskning/forskningscentrum/virtuellasystem/ingame-lab/projects---ingame-lab

[6] Benveniste, S., Jouvelot, P. and Michel, R. Wii Game Technology for Music Therapy: A First Experiment with Children Suffering from Behavioral Disorders. CCIS'08, IADIS Gam. Conf., Amsterdam, July 2008.

[7] Benveniste, S. www.cri.ensmp.fr/ sbenven

[8] Bruscia, K. Case Studies in Music Therapy. Barcelona Publishers, Barcelona (ES), 1991.

[9] Bruscia, K. Improvisational Models of Music Therapy. Charles C. Thomas, Springfield, IL (US), 1987.
[10] Chouvier, B. Les processus psychiques de la médiation : créativité et travail thérapeutique. Dunod, Paris (FR), 2002.

[11] Denis, G., and Jouvelot, P. Motivation-Driven Educational Game Design: Applying Best Practices to Music Education. ACM SIGCHI Advances in Computer Education Conference, Valencia, June 2005.

[12] Erkkilä, J., Lartillot, O., Luck, G., Riikkilä, K., Toiviainen, P. Intelligent Music Systems in Music Therapy. Music Therapy Today (online) Vol. V, Issue 5.

[13] HopeLab Inc. www.re-mission.net

[14] Hunt, A., Kirk, R., and Neighbour, M. Multiple Media Interfaces for Music Therapy. IEEE Multimedia Special Issue on Multisensory Communication, July - September, 2004, 50-58.

[15] IGN Wii. uk.wii.ign.com/objects/827/827335.html

[16] Kahol, K. and Smith, M. presented in Reilly, M. A Wii Warm-up Hones Surgical Skills. www.newscientist.com/article/mg19726396.100-a-wiiwarmup-hones-surgical-skills.html

[17] Kenner, C. carl.kenner.googlepages.com/glovepie

[18] Lecourt, E. La musicothérapie analytique de groupe. Editions Fuzeau, Courlay (FR), 2007.

[19] Lewin, K. Action Research and Minority Problems. Journal of Social Issues 2, 4 (1946), 34-46.

[20] Somers, R. Video demo of Wiidrums. www. youtube.com/watch?v=8FftLxuYzRA

[21] Stora, M. Histoire d'un atelier jeu vidéo : Ico, un conte de fée interactif pour des enfants en manque d'interactions. www.omnsh.org/spip.php?article84

[22] Streeter, E. www.musictherapylogbook.com

[23] Streeter, E. Reactions and Responses from the Music Therapy Community to the Growth of Computers and Technology - Some Preliminary Thoughts. Voices online, Volume 7, March 2007.

[24] Zhou, R. Feature Extraction of Musical Content for Automatic Music Transcription. Ph.D. Thesis, Ecole polytechnique fédérale de Lausanne, 2006 